\title{
(2) OPEN ACCESS \\ Parental perspective on important health outcomes of extremely preterm infants
}

\author{
Magdalena Jaworski, ${ }^{1,2}$ Annie Janvier, ${ }^{1,2,3,4,5,6,7}$ Claude Julie Bourque, 2,3,5 \\ Thuy-An Mai-Vo, ${ }^{5}$ Rebecca Pearce, ${ }^{6}$ Anne R Synnes (D) , ${ }^{8}$ Thuy Mai Luu (D) ${ }^{1,5}$
}

\begin{abstract}
- Additional supplemental material is published online only. To view, please visit the journal online (http://dx.doi. org/10.1136/archdischild2021-322711)
\end{abstract}

For numbered affiliations see end of article.

\section{Correspondence to}

Dr Thuy Mai Luu, Pediatrics and Research Center, Centre Hospitalier Universitaire SainteJustine, Montreal, QC H3T 1C5,

Canada;

thuy.mai.luu@umontreal.ca

Received 22 June 2021 Accepted 3 November 2021

\section{Check for updates}

(C) Author(s) (or their employer(s)) 2021. Re-use permitted under CC BY-NC. No commercial re-use. See rights and permissions. Published by BMJ.

To cite: Jaworski M, Janvier A, Bourque CJ, et al. Arch Dis Child Fetal Neonatal Ed Epub ahead of print: [please include Day Month Year]. doi:10.1136/archdischild2021-322711

\section{ABSTRACT \\ Background and objective Neonatal outcome research and clinical follow-up principally focus on neurodevelopmental impairment (NDI) after extremely preterm birth, as defined by the scientific community, without parental input. This survey aimed to investigate parental perspectives about the health and development of their preterm children. \\ Methods Parents of children aged 18 months to 7 years born $<29$ weeks' gestational age presenting at a neonatal follow-up clinic over a 1-year period were asked to evaluate their children's health and development. They were also asked the following question: 'if you could improve two things about your child, what would they be?' Responses were analysed using mixed methods. Logistic regressions were done to compare parental responses.}

Results 248 parents of 213 children (mean gestational age 26.6 \pm 1.6 weeks, 20\% with severe NDI) were recruited. Parents evaluated their children's health at a median of 9/10. Parental priorities for health improvements were (1) development, mainly behaviour, emotional health and language/communication (55\%); (2) respiratory heath and overall medical fragility $(25 \%)$; and (3) feeding/growth issues (14\%). Nineteen per cent explicitly mentioned 'no improvements'. Parents were more likely to state 'no improvements' if child had no versus severe NDI OR $4.33(95 \% \mathrm{Cl} 1.47$ to 12.75$))$ or if parents had no versus at least a high school diploma (OR 4.01 (95\% 1.99 to 8.10)).

Conclusions Parents evaluate the health of their preterm children as being very good, with positive perspectives. Parental concerns outside the developmental sphere should also be addressed both in clinical follow-up and research.

\section{INTRODUCTION}

Preterm birth is associated with increased risk of neurosensory impairment and developmental delay. ${ }^{1-3}$ The systematic assessment of these aspects of development are combined and categorised by levels of neurodevelopmental impairment (NDI). ${ }^{4}$ To monitor neurodevelopmental outcomes, neonatal follow-up programmes have become the standard of care. ${ }^{4-6}$ Neonatal follow-up research has thus been shaping the landscape of prematurity outcomes for several decades, determining which outcomes are reported. Classification of the outcomes of preterm children into NDI categories is now the norm in research. ${ }^{7}$ However, less interest is directed towards whether these outcomes are also of importance to families. ${ }^{8}$ This often

\section{What is already known on this topic?}

- Preterm outcome research generally focuses on adverse health and neurodevelopmental rates.

- Research results are used to council parents yet parental opinions regarding what outcomes are important for them has not been studied.

\section{What this study adds?}

Parents of extremely preterm children report a balanced perspective on their child.

- This includes positive traits as well as concerns which encompass development and medical fragility, nutrition and respiratory health.

- Concerns vary with child's neurodevelopment, age and parental education.

leads to the erroneous perception that some children have a 'bad' or 'good' outcome, only based on NDI. Furthermore, children categorised as having 'normal' outcomes/no disability may have problems that are overlooked. ${ }^{9} 10$ The reporting of outcomes pertaining to the health and development of preterm children is undoubtedly essential, but could benefit from adequate representation and framing when it comes to families. ${ }^{11} 12$

Researchers across medical fields are increasingly reporting patient-important outcomes. Initiative such as those reported by the Patient-Centered Outcomes Research Institute are thought to help determine outcomes that patients find most important, as they represent their lived experience with illness. ${ }^{13}$ The aim of the current study was to expand the knowledge of parental/family perspectives regarding the health of their children born preterm.

\section{METHODS}

\section{Design and study participants}

The Parents' Voices Project is a cross-sectional survey of parents of extremely preterm children seen at a level-III university hospital neonatal follow-up clinic. All infants born $<29$ weeks' gestational age (GA) are eligible for follow-up after discharge from the neonatal intensive care unit (NICU) for neurodevelopmental monitoring. All children are seen at 18 months corrected age (CA), at 36 months CA, 
then at 5 years and 7 years, according to standardised follow-up protocols (online supplemental table s1). ${ }^{1}$

Between 1 July 2018 and 31 July 2019, parents of preterm children who were scheduled for a neonatal follow-up clinic visit were approached to participate. Foster parents were excluded.

\section{Study procedure}

At least one parent per eligible child was sent an email invitation to participate in the study. If parents did not respond, a research assistant attempted to contact them by telephone or in person at the follow-up visit. They were offered to respond to the questionnaire prior to the follow-up visit either online, on paper or by interview.

The questionnaire was developed and reviewed in partnership with parent stakeholders. Parents were asked to rate their child's health on a scale of 0 (very poor) to 10 (excellent). They were then asked to answer the following question: 'If you could improve any two things about your child's health and/or development, what would they be?' When possible, both parents were invited to participate separately. Parents of twins or triplets were asked to complete one questionnaire per child.

\section{Demographic data and developmental outcomes}

Parent characteristics including self-reported race, highest level of education and family composition were retrieved from questionnaires administered to all families during clinical care. Neonatal characteristics and demographics were collected from the infant's medical record by trained research personnel using Canadian Neonatal Network abstractor manual definitions. ${ }^{14}$ Neurodevelopmental outcome was determined for each child at the standardised 18-month CA visit following the Canadian Neonatal Follow-Up Network protocol. ${ }^{4}$ In summary, all children were administered the Bayley Scales of Infant and Toddler Development, 3rd edition (Bayley III). A neurological examination was performed by a paediatrician to identify signs of cerebral palsy and determine functional level using the Gross Motor Functional Classification System (GMFCS). ${ }^{15}$ Finally, data on visual and hearing function were retrieved from the medical chart. A level of neurodevelopmental impairment (NDI) was assigned for each child based on the 18-month assessment (table 1). ${ }^{4}$

\section{Data analyses}

Descriptive statistics were used to describe the study population and parental evaluations of their children's health. When both parents provided a rating for a single child, the mean score was calculated. Comparison of parental rating score in relation to NDI status was done using the Kruskal-Wallis test.

Answers to the open-ended question were analysed using descriptive content analysis. Themes were developed simultaneously and independently by a team of eight investigators representing neonatology, general paediatrics, developmental paediatrics, rehabilitation care, sociology and parents. During the initial exploration of data, codes, subcodes, and coding definition and structure were developed gradually until consensus was reached. Then, systematic coding of all parental answers was done in an independent fashion by rotating teams of two investigators and discrepancies were resolved with a third coder. This brought multiple perspectives to the analysis to prevent mistakes and individual biases; all data were reviewed by a parent in initial or final coding steps. Codes of the qualitative content were introduced in the database as dichotomised variables (Yes/ No). Statistical analyses were conducted with SPSS (IBM SPSS Statistics V.24). Logistic regressions were done to calculate the frequency of each theme in relation to the child's age, gestational age, level of NDI (none, mild to moderate, severe) and parental characteristics. As these analyses were exploratory in nature, we did not adjust for multiple comparisons.

\section{RESULTS}

During the study period, at least one parent for the 258 children scheduled for follow-up were contacted either by email, telephone or in person (figure 1); 248 parents of 213 children (83\% of eligible children) participated and provided 285 individual responses. Both parents answered the questionnaire for 71 children; there were only maternal answers for 128 children and only paternal answers for 14 . Among responding mothers and fathers, $63 \%$ and $82 \%$, respectively, self-identified as Caucasian and $82 \%$ and $87 \%$, respectively, reported having at least a high school diploma (table 2).

The 213 children were born at a mean gestational age of 26.6 weeks with mean birth weight of $907 \mathrm{~g}$ (table 3). For the majority (59\%) of participants, parents answered the questionnaire at the 18-month or 36-month CA visit. Based on the 18-month assessment, $55 \%$ met criteria for no NDI, $25 \%$ had a mild to moderate NDI and $20 \%$ a severe NDI.

\section{Evaluation of health}

For the 213 children, the median score for health as reported by parents was 9/10 (IQR 7.5-10, range 3-10). When both parents responded for the same child, agreement was strong (Pearson's $\mathrm{r}=0.668$ ). For children with no NDI, mild to moderate NDI,

Table 1 Definition of neurodevelopmental impairment

\begin{tabular}{llll}
\hline Impairments & $\begin{array}{l}\text { Severe neurodevelopmental impairment (any } \\
\text { one or more of the following)* }\end{array}$ & $\begin{array}{l}\text { Mild-moderate neurodevelopmental impairment } \\
\text { (any one or more of the following) }\end{array}$ & No neurodevelopmental impairment \\
\hline Motor & CP with GMFCS 3,4 or 5 & CP with GMFCS 1 or 2 & No cerebral palsy \\
& Bayley III Motor Composite $<70$ & Bayley III Motor Composite $70-84$ & Bayley III Motor Composite $\geq 85$ \\
Cognitive & Bayley III Cognitive Composite $<70$ & Bayley III Cognitive Composite 70-84 & Bayley III Cognitive Composite $\geq 85$ \\
Language & Bayley III Language Composite $<70$ & Bayley III Language Composite 70-84 & Bayley III Language Composite $\geq 85$ \\
Hearing & Hearing aid or cochlear implant & $\begin{array}{l}\text { Sensorineural/mixed hearing loss without requirement } \\
\text { for hearing devices }\end{array}$ & No hearing loss as per audiology report \\
Vision & Bilateral visual impairment & Unilateral visual impairment & $\begin{array}{l}\text { No visual impairment as per ophthalmology } \\
\text { report }\end{array}$ \\
\hline
\end{tabular}

*Includes children who could not be tested using the Bayley-III but who had a Bayley-III Adaptive Behaviour score $<70$ or who were assessed to have a significant developmental delay.

tChildren who could not be tested using the Bayley-III but who had a Bayley-III Adaptive Behaviour score between 70 and 84.

CP, cerebral palsy; GMFCS, Gross Motor Function Classification System. 
Original research

\section{8 patients aged 18 months CA to 7 years eligible for} follow-up visit between July 2018-July 2019

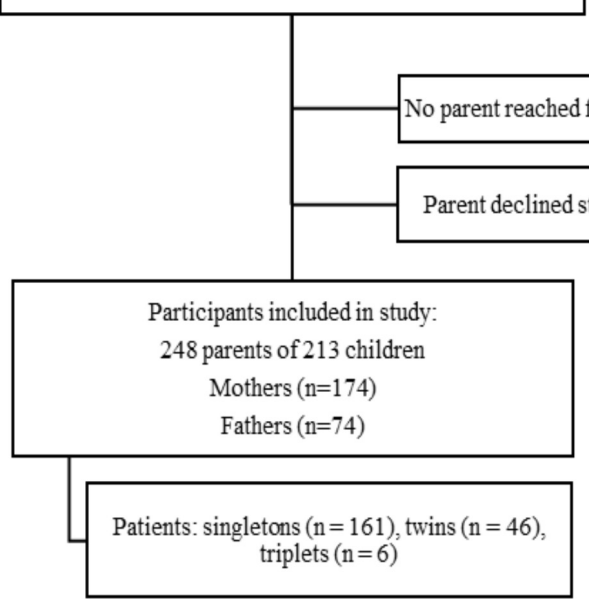

Figure 1 Flowchart of study population.

and severe NDI, the median health score was 9 (IQR 8.5-10), 8 (IQR 7-9) and 7 (IQR 6-9), respectively $(p<0.001)$. We examined children whose parent(s) rated their health as 6 or less ( $\leq 10$ th percentile of the cohort, $n=28$ ): 4 had no NDI, 9 mild to moderate NDI, and 15 severe NDI.

\section{Parental priorities for health improvements}

The main themes identified included (1) development (55\%), (2) respiratory health and fragility (25\%), (3) nutrition/feeding and growth problems (14\%), with (4) 19\% $(n=52)$ of parents reporting there was nothing to improve (figure 2 ). When parents described their child's development as suboptimal, language and communication (19\%), behaviour and emotional health (18\%), motor development and movements $(15 \%)$ as well as cognitive and learning skills (14\%) were most often cited as top concerns. Table 4 shows parents describing a wide array of problems.

\section{Association between parent and child characteristics and parental priorities}

Figure 3A shows a comparison of themes reported by parents of preterm children by NDI category (online supplemental tables s2 and s3). Development was mentioned equally by all parents. Growth and feeding were more concerning to parents of children in the severe NDI group, as compared with the no-NDI group (OR 2.86; 95\% CI 1.27 to 6.45 ). Respiratory illness and

\begin{tabular}{|c|c|c|}
\hline & $\begin{array}{l}\text { Mothers } \\
n=174\end{array}$ & $\begin{array}{l}\text { Fathers } \\
n=74\end{array}$ \\
\hline Mean age at birth (SD), years & $32(5)$ & NA \\
\hline Lone parent, n (\%) & 14/172 (8) & $1 / 74(1)$ \\
\hline \multicolumn{3}{|l|}{ Self-reported race, n (\%) } \\
\hline Caucasian & 108/171 (63) & $59 / 72(82)$ \\
\hline Black & $42 / 171(25)$ & $7 / 72(10)$ \\
\hline Other & $21 / 171(12)$ & $6 / 72(8)$ \\
\hline Education $\geq$ high school, n (\%) & $138 / 168(82)$ & $62 / 71(87)$ \\
\hline No of responses provided & 199 & 86 \\
\hline Singleton & $139(70 \%)$ & $60(70 \%)$ \\
\hline Twin or triplet & $60(30 \%)$ & $26(30 \%)$ \\
\hline
\end{tabular}

\begin{tabular}{ll}
\hline Table 3 Child characteristics $(\mathrm{n}=213)$ & \\
\hline Mean gestational age (SD), weeks & $26.6(1.6)$ \\
\hline Mean birth weight (SD), $\mathrm{g}$ & $907(247)$ \\
Male, $\mathrm{n}(\%)$ & $106(50)$ \\
\hline Multiple births, $\mathrm{n}(\%)$ & $65(31)$ \\
Severe brain injury ${ }^{*}, \mathrm{n}(\%)$ & $44(21)$ \\
Bronchopulmonary dysplasiatt, $\mathrm{n}(\%)$ & $130 / 203(64)$ \\
Median duration of NICU stay (IQR), days & $105(78,130)$ \\
NDI at 18 months CA, $\mathrm{n}(\%)$ & \\
None & $114 / 209(55)$ \\
Mild/moderate & $53 / 209(25)$ \\
Severe & $42 / 209(20)$ \\
Mean Bayley-III scores at 18 months CA (SD) & \\
Cognition & $94(14)$ \\
Language & $91(16)$ \\
Motor & $91(14)$ \\
Current age, $\mathrm{n}(\%)$ & \\
18 months CA & $65(31)$ \\
36 months CA & $59(28)$ \\
5 years & $56(26)$ \\
7 years & $33(16)$ \\
\hline
\end{tabular}

*Severe brain injury defined as grade 3-4 intraventricular haemorrhage, cystic periventricular leucomalacia, cerebellar haemorrhage.

tBronchopulmonary dysplasia defined as oxygen use at 36 weeks of postmenstrual age.

$\mathrm{CA}$, corrected age; NDI, neurodevelopmental impairment; $\mathrm{NICU}$, neonatal intensive care unit.

fragility were more frequently reported as priorities by parents of children in the mild-moderate NDI category, as compared with the severe NDI category (OR 2.50; 95\% CI 1.04 to 6.00). Finally, parents of children without NDI were more likely to report having nothing to improve compared with parents in

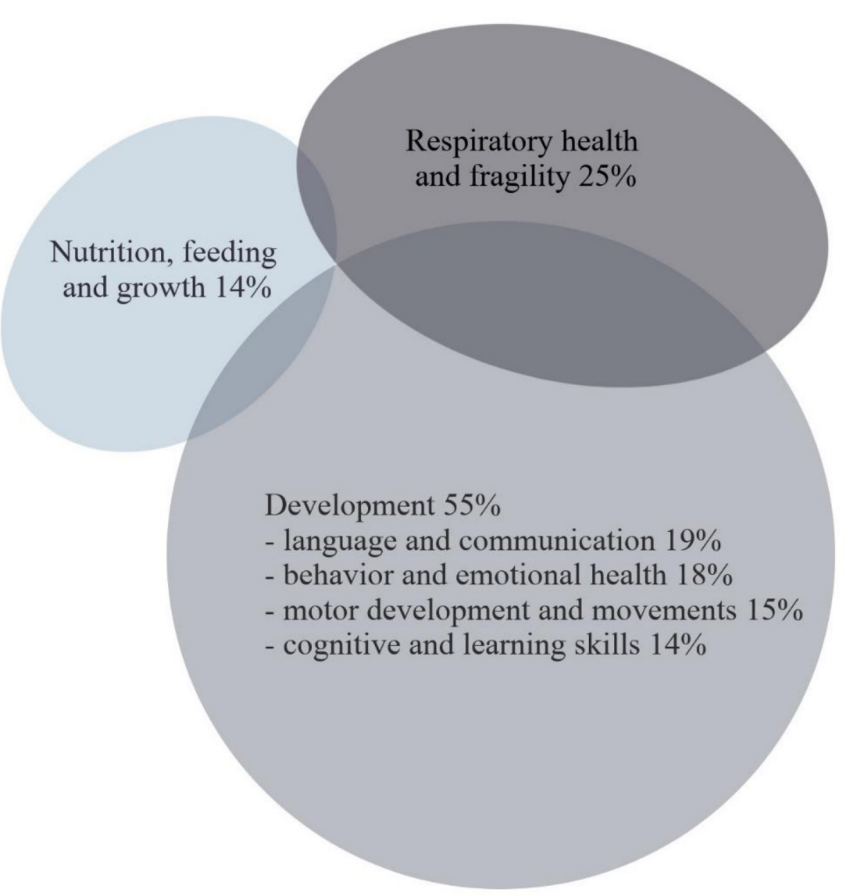

Figure 2 Venn diagram of parental priorities for improvements. Some parents reported on several themes, which is illustrated by the overlapping areas. 
Table 4 Examples of quotes provided by parents

\begin{tabular}{|c|c|c|}
\hline \multirow[t]{4}{*}{ Development } & "Her language, I wish she would express herself more clearly." & Mother of a 3-year-old girl born at 26 weeks \\
\hline & "A better management of his crises (when he gets out of control)." & Father of 5-year-old born at 27 weeks \\
\hline & "She acts like a baby, I'm worried about school." & Mother of a 3-year-old girl born at 26 weeks \\
\hline & "His gross motor skills, including running." & Mother of a 3-year-old boy born at 27 weeks \\
\hline \multirow[t]{3}{*}{ Respiratory health and fragility } & "His lungs are still fragile." & Mother of a 5-year-old boy born at 25 weeks \\
\hline & "His immune system, his pneumonias, he is sick all the time." & Mother of an 18-month-old boy born at 27 weeks \\
\hline & "Inflammation of his lungs every time he catches a virus." & Father of 18 -month-old boy born at 28 weeks \\
\hline \multirow[t]{3}{*}{ Growth and nutrition } & "I hope she will learn how to eat." & Father of an 18-month-old girl born at 25 weeks \\
\hline & "Remove her gastrostomy." & Mother of an 18-month-old girl born at 23 weeks \\
\hline & "His reflux and frequent vomiting. He does not eat much." & Father of 5-year-old boy born at 25 weeks \\
\hline
\end{tabular}

the two other groups. Figure 3B compares parental responses according to their child's age (online supplemental tables s 2 and s3). Parents of 18-month-old children were less likely to mention development as an area for improvement. Parents of children aged 18-36 months were more likely to express concerns regarding respiratory health, compared with the 7 -year-old group, and growth and nutrition issues compared with parents of older children.

There was no difference between mothers and fathers or by gestational age groups (22-24 weeks, 25-26 weeks, 27-28 weeks) with respect to the frequency of themes invoked by parents (online supplemental tables s2 and s3). When examining

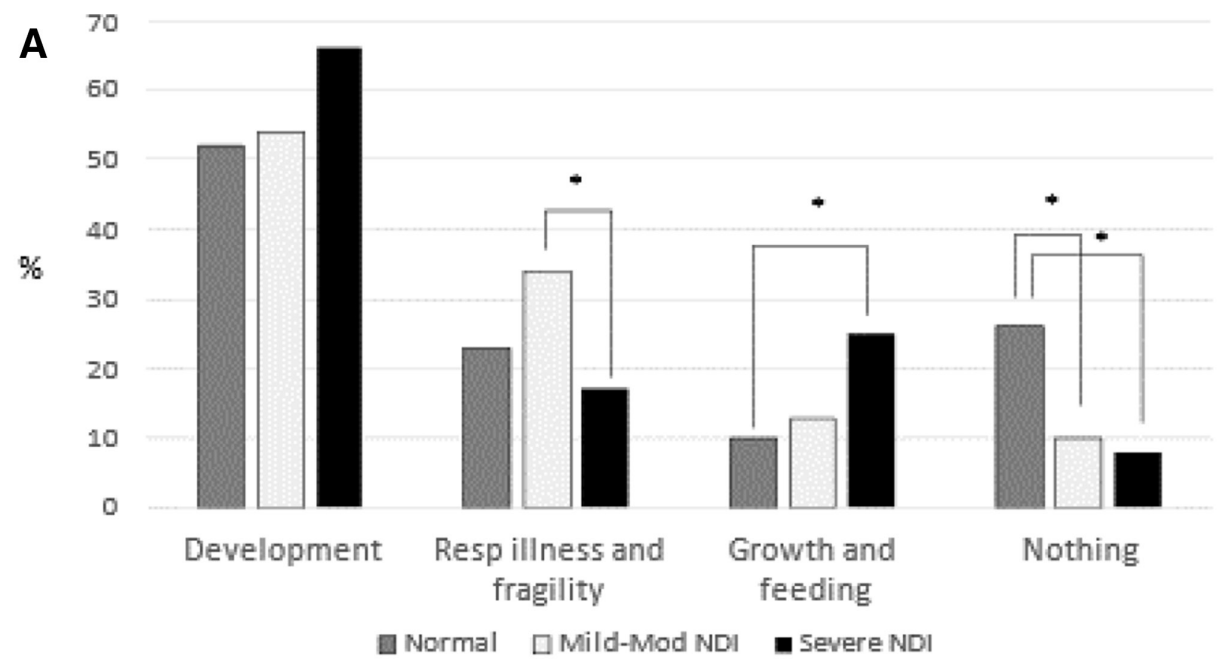

B

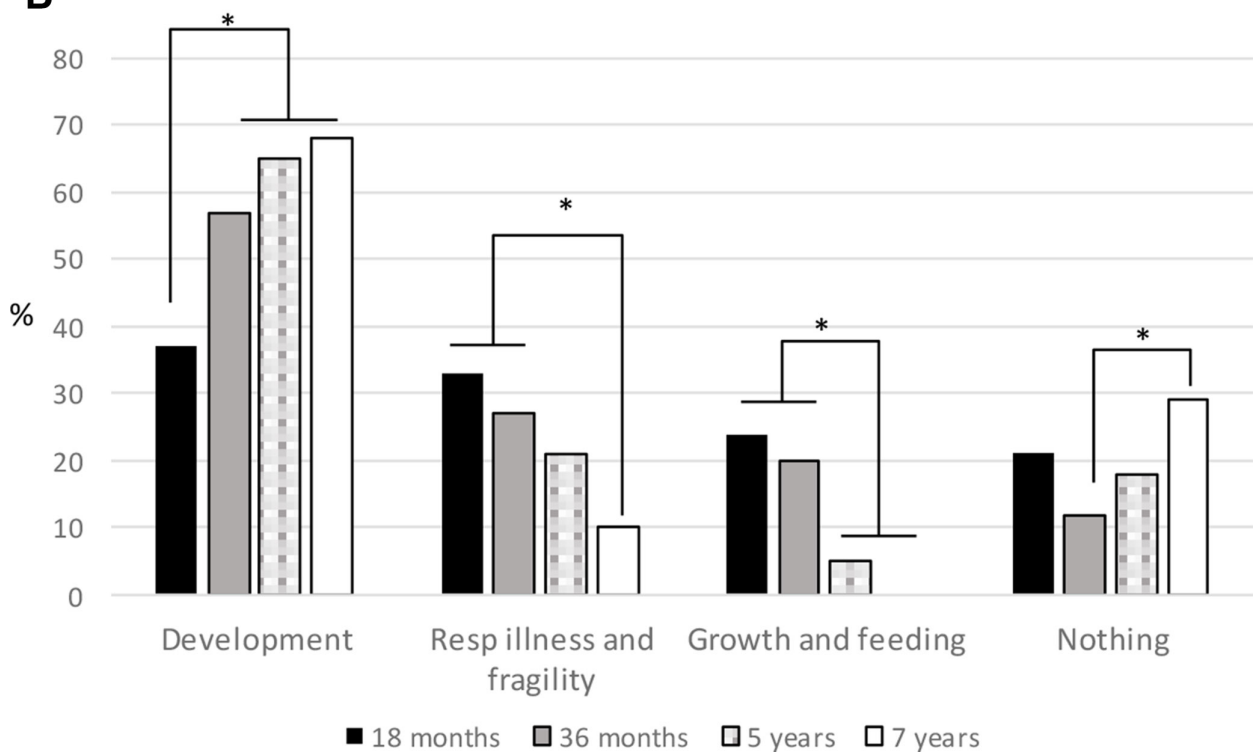

Figure 3 Areas of improvements by (A) neurodevelopmental impairment, (B) child age. NDI, neurodevelopmental impairment. * $\mathrm{p}<0.05$ by bivariate logistic regression. 
responses in relation to level of education, parents who had not completed high school were less likely to report wishing to improve their child's development compared with parents with at least a high school diploma (OR $0.46,95 \%$ CI 0.24 to 0.88 ). They were also more likely to respond that nothing needed to change (OR 4.01; 95\% CI 1.99 to 8.10 ).

\section{DISCUSSION}

Measuring and classifying the outcomes of preterm infants is important for a number of reasons: identifying health and developmental concerns to ensure that children receive optimal healthcare support, to improve healthcare practices for all children (audit and quality improvement), for research purposes and to inform parents about prognostic elements regarding their preterm children. ${ }^{4-6}$ This is the first study investigating how parents rate their children's health and also their concerns and priorities: what they wished they could improve in an openended fashion (without an a priori). Parents rated their child's overall health as being high, despite disability, even if the presence and severity of NDI influenced parents' rating. In addition, parents did acknowledge the challenges faced by their children, mentioning several areas where improvements were desired.

These high ratings, despite the hardship of the NICU experience, overall fragility and, for some children, disability, potentially reflect the resilience of families and positive transformations. ${ }^{16}$ In other studies, parents reported a high quality of life for their children despite disabilities or described their child's happiness and good personality. ${ }^{17} 18$ These findings may indicate that for families, the well-being of their child, as opposed to disability, is what is truly important when health is evaluated.

Because we wanted to capture parents' subjective evaluation of their child, we did not use standardised health-related quality of life questionnaires. Other authors have previously reported the high parent-perceived and self-perceived quality of life ratings. ${ }^{17} 19$ In our cohort, perceived health remains high, but decreases with level of NDI, suggesting that neurodevelopmental outcomes do have an impact on parental subjective evaluations. In previous studies, authors have found that adults born premature rate their quality of life highly, independent of NDI. However, they are aware of their health and functional limitations. ${ }^{20}$ Perhaps the parents in our study had a similar viewing of their child's health: lucidly assessing their child's function and what their children could do, and not only their diagnoses and limitations.

Even though parents generally rate their child's health with high scores, there is always room for improvement. This should be the basis for ongoing neonatal follow-up research as well as clinical research at large. In the clinical setting, health practitioners screen for difficulties early on, and the focus is mainly on child development. Development (especially behaviour and emotions) and respiratory health are the main areas parents wished they could improve. Some adverse outcomes considered to be mild by the medical literature, such as behavioural problems, hyperactivity and emotional health, were often considered as major problems by families. Whether these problems have more negative impacts on the child as well as the whole family, as opposed to deafness or using a walker due to cerebral palsy (classified as severe NDI), is a possibility. Interestingly, in terms of development, as children grew older, parents were more likely to express concerns about language and communication, socioemotional problems and learning difficulties. Yet, standardised follow-up in most high-income countries often stops after 18-36 months CA, which might be too early to uncover more subtle neurodevelopmental dysfunctions, such as those reported by parents, that are likely to interfere with school achievement. ${ }^{21}$ In a recent European survey, only $27 \%$ of very preterm children were receiving follow-up care at 5 years. ${ }^{22}$

Furthermore, as previously shown, children of lower socioeconomic status were less likely to attend follow-up. ${ }^{23}{ }^{24}$ Despite the association between lower socioeconomic status and neurodevelopmental vulnerabilities, especially in children at higher biological risk, ${ }^{25}$ a lower level of parental education, in our study, was associated with parents explicitly or implicitly responding that nothing needed to be improved with their child. This could be interpreted as accepting the child as is, regardless of their challenges. This could also be associated with parental expectations as related to their own achievements, which may impact the value they place on educational achievements. ${ }^{26}$ This raises the importance of engaging parents across educational levels to ensure proper developmental surveillance and screening so timely referral to intervention services is done when needed.

Our study also underlines the importance of reporting and investigating other outcomes, such as respiratory health and feeding/nutrition difficulties, as these are very important to many families, especially with younger children. These items should be systematically included and addressed in neonatal follow-up research, as they have a major impact on families' daily lives and function and for some families, they represent the main disability their children have.$^{27}$

This study did not have a comparison group of full-term children, whose parents would certainly also have concerns regarding health and development. However, we wished to examine the health and developmental outcomes which are of specific importance to parents of children born extremely premature. Also, we did not use standardised health-related quality of life questionnaires. This would have not allowed us to identify outcomes of interest to parents in their own wordswhat is most important to them-and can only be achieved by asking open-ended questions. Among parents of children who came for follow-up, the response rate was high (98\%), but these responses represented a selected group. Given that preterm children attending follow-up are usually at higher biological risk of medical or developmental problems, ${ }^{28}$ it is possible that we have underestimated the percentage of parents who actually did not wish to improve anything with their children. We are confident that parent and family important outcomes in this article represent the perspectives of parents in our centre, and possibly in places where children have access to universal healthcare and systematic follow-up. On the other hand, parent/family important outcomes may be different in centres where systematic follow-up or access to early intervention are not available.

\section{CONCLUSION}

Parents evaluate the health of their preterm children as being very good, with positive perspectives. Many express concerns outside the developmental sphere, such as medical fragility, nutrition and respiratory health. Many also express concerns about emotions and behaviour, which are not addressed nor categorised optimally in follow-up studies. Priorities differ according to the child's level of NDI, age and parental level of education. Incorporating measures reflecting what parents view as important can ensure that research findings are meaningful to families and that clinical follow-up targets relevant issues.

\section{Author affiliations}

'Department of pediatrics, Centre Hospitalier Universitaire Sainte-Justine, Montreal, Québec, Canada 
${ }^{2}$ Clinical Ethics Unit, Centre Hospitalier Universitaire Sainte-Justine, Montreal, Québec, Canada

${ }^{3}$ Clinical Ethics and Family Partnership Research Unit, CHU Sainte-Justine Centre de Recherche, Montreal, Québec, Canada

${ }^{4}$ Palliative care unit, Centre Hospitalier Universitaire Sainte-Justine, Montreal, Québec, Canada

${ }^{5}$ Centre Hospitalier Universitaire Sainte-Justine Research Center, Montreal, Québec, Canada

${ }^{6}$ Bureau du partenariat patients-familles-soignants, Centre Hospitalier Universitaire Sainte-Justine, Montreal, Québec, Canada

${ }^{7}$ Bureau de l'éthique clinique, Université de Montréal, Montreal, Québec, Canada

${ }^{8}$ British Columbia Children's Hospital Research Institute, Vancouver, British Columbia, Canada

Acknowledgements We would like to thank Ms Kate Robson, from the Canadian Premature Babies Foundation, Dr Paige Therrien Church, Ms Lindsay Richter, Dr Émilie Thivierge, Dr Laurie-Anne Duquette, Dr Aurélie Fortin and all participating families for their unique contribution to this work.

Contributors MJ conceptualised and designed the study, performed thematic analysis and drafted the initial manuscript. AJ conceptualised and designed the study, obtained funding, conducted the study, participated in thematic analysis, and reviewed and revised the manuscript. CJB conceptualised and designed the study, obtained funding, conducted the study, participated in thematic analysis, and reviewed and revised the manuscript. T-AM-V collected data, participated in thematic analysis, and reviewed and revised the manuscript. RP conducted the study, participated in thematic analysis, and reviewed and revised the manuscript. ARS conceptualised and designed the study, obtained funding, and reviewed and revised the manuscript. TML conceptualised and designed the study, obtained funding, conducted the study, performed thematic analysis and drafted the initial manuscript. TML is the author acting as guarantor. All authors approved the final manuscript as submitted and agree to be accountable for all aspects of the work.

Funding This study is funded by the CHILD-BRIGHT Network, with funding from the Canadian Institutes of Health Research (CIHR) under the Strategy for PatientOriented Research (SPOR) grant number SCA-145104. AJ and TML have salary support from the Fonds de Recherche en Santé du Québec.

Competing interests None declared.

Patient consent for publication Not applicable.

Ethics approval The study protocol was approved by Centre Hospitalier Universitaire Sainte-Justine (Montreal, Canada) institutional review board (Number 2019-1950), and all parents signed a consent form.

Provenance and peer review Not commissioned; externally peer reviewed.

Data availability statement The data that support the findings of this study are available from the corresponding author (TML) upon reasonable request.

Supplemental material This content has been supplied by the author(s). It has not been vetted by BMJ Publishing Group Limited (BMJ) and may not have been peer-reviewed. Any opinions or recommendations discussed are solely those of the author(s) and are not endorsed by BMJ. BMJ disclaims all liability and responsibility arising from any reliance placed on the content. Where the content includes any translated material, BMJ does not warrant the accuracy and reliability of the translations (including but not limited to local regulations, clinical guidelines, terminology, drug names and drug dosages), and is not responsible for any error and/or omissions arising from translation and adaptation or otherwise.

Open access This is an open access article distributed in accordance with the Creative Commons Attribution Non Commercial (CC BY-NC 4.0) license, which permits others to distribute, remix, adapt, build upon this work non-commercially, and license their derivative works on different terms, provided the original work is properly cited, appropriate credit is given, any changes made indicated, and the use is non-commercial. See: http://creativecommons.org/licenses/by-nc/4.0/.

ORCID iDs

Anne R Synnes http://orcid.org/0000-0002-6917-0978

Thuy Mai Luu http://orcid.org/0000-0003-2222-186X

\section{REFERENCES}

1 Doyle LW, Anderson PJ, Battin M, et al. Long term follow up of high risk children: who, why and how? BMC Pediatr 2014;14:279.

2 Maitre NL. Neurorehabilitation after neonatal intensive care: evidence and challenges. Arch Dis Child Fetal Neonatal Ed 2015;100:F534-40.

3 Broyles RS, Tyson JE, Heyne ET, et al. Comprehensive follow-up care and lifethreatening illnesses among high-risk infants: a randomized controlled trial. JAMA 2000;284:2070-6.

4 Synnes A, Luu TM, Moddemann D, et al. Determinants of developmental outcomes in a very preterm Canadian cohort. Arch Dis Child Fetal Neonatal Ed 2017;102:F23 5-F234.

5 Fanaroff AA, Hack M, Walsh MC. The NICHD neonatal research network: changes in practice and outcomes during the first 15 years. Semin Perinatol 2003;27:281-7.

6 Sauve R, Lee SK. Neonatal follow-up programs and follow-up studies: historical and current perspectives. Paediatr Child Health 2006;11:267-70.

7 Stoll BJ, Hansen NI, Bell EF, et al. Trends in care practices, morbidity, and mortality of extremely preterm neonates, 1993-2012. JAMA 2015;314:1039-51.

8 Bell EF, Rysavy MA. What parents want to know after preterm birth. J Pediatr 2018;200:10-11.

9 Janvier A, Farlow B, Baardsnes J, et al. Measuring and communicating meaningful outcomes in neonatology: a family perspective. Semin Perinatol 2016;40:571-7.

10 Adams-Chapman I, Bann CM, Vaucher YE, et al. Association between feeding difficulties and language delay in preterm infants using Bayley Scales of Infant Development-Third Edition. J Pediatr 2013;163:680-5.

11 Rysavy MA, Marlow N, Doyle LW, et al. Reporting outcomes of extremely preterm births. Pediatrics 2016;138. doi:10.1542/peds.2016-0689. [Epub ahead of print: 11 08 2016].

12 Kilbride HW, Aylward GP, Doyle LW, et al. Prognostic neurodevelopmental testing of preterm infants: do we need to change the paradigm? J Perinatol 2017;37:475-9.

13 Mehran R, Baber U, Dangas G. Guidelines for patient-reported outcomes in clinical trial protocols. JAMA 2018;319:450-1.

14 Squires J, Bricker D, Twombly E. Ages \& Stages Questionnaires: Social-Emotional (ASQ: SE). Baltimore, MD: Paul H. Brookes Publishing Co, 2002.

15 Rosenbaum PL, Palisano RJ, Bartlett DJ, et al. Development of the gross motor function classification system for cerebral palsy. Dev Med Child Neuro 2008; $50: 249-53$

16 Scorgie K, Sobsey D. Transformational outcomes associated with parenting children who have disabilities. Ment Retard 2000;38:195-206.

17 Saigal S, Rosenbaum PL, Feeny D, et al. Parental perspectives of the health status and health-related quality of life of teen-aged children who were extremely low birth weight and term controls. Pediatrics 2000;105:569-74.

18 Jaworski M, Janvier A, Lefebvre $F$, et al. Parental perspectives regarding outcomes of very preterm infants: toward a balanced approach. J Pediatr 2018;200:58-63.

19 Saigal S, Stoskopf B, Pinelli J, et al. Self-perceived health-related quality of life of former extremely low birth weight infants at young adulthood. Pediatrics 2006;118:1140-8.

20 Zwicker JG, Harris SR. Quality of life of formerly preterm and very low birth weight infants from preschool age to adulthood: a systematic review. Pediatrics 2008; 121:e366-76.

21 Johnson S, Marlow N. Early and long-term outcome of infants born extremely preterm. Arch Dis Child 2017;102:97-102.

22 Seppänen A-V, Draper ES, Petrou S, et al. Follow-up after very preterm birth in Europe. Arch Dis Child Fetal Neonatal Ed 2021:fetalneonatal-2020-320823.

23 Castro L, Yolton K, Haberman B, et al. Bias in reported neurodevelopmental outcomes among extremely low birth weight survivors. Pediatrics 2004;114:404-10.

24 Ballantyne M, Stevens B, Guttmann A, et al. Maternal and infant predictors of attendance at neonatal follow-up programmes. Child Care Health Dev 2014:40:250-8.

25 Taylor CL, Christensen D, Stafford J, et al. Associations between clusters of early life risk factors and developmental vulnerability at age 5 : a retrospective cohort study using population-wide linkage of administrative data in Tasmania, Australia. BMJ Open 2020;10:e033795-e.

26 Janvier A. "Pepperoni pizza and sex". Curr Probl Pediatr Adolesc Health Care 2011;41:106-8.

27 Howe T-H, Sheu C-F, Wang T-N, et al. Parenting stress in families with very low birth weight preterm infants in early infancy. Res Dev Disabil 2014;35:1748-56.

28 Guillén U, DeMauro S, Ma L, et al. Relationship between attrition and neurodevelopmental impairment rates in extremely preterm infants at 18 to 24 months: a systematic review. Arch Pediatr Adolesc Med 2012;166:178-84. 